\title{
Optical detection of single transparent nanoparticles
}

\author{
Yuri Vainer ${ }^{1, *}$, Alexander Zybin ${ }^{2}$, Nikolay Gippius ${ }^{3}$, and Anastasia Malek $^{4}$ \\ ${ }^{1}$ Institute of Spectroscopy RAS, 142190 Moscow, Troitsk, Russia \\ ${ }^{2}$ Leibniz-Institut für Analytische Wissenschaften, 44139 Dortmund, Germany \\ ${ }^{3}$ Skolkovo Institute of Science and Technology, 121205 Moscow, Russia \\ ${ }^{4}$ N.N. Petrov Institute of Oncology, 197758 Saint-Petersburg, Russia
}

\begin{abstract}
We will present the novel experimental far-field optical microscopy technique for detection of single nanoparticles, which weakly absorb a visible light and are characterized by refractive index close to its value in nearby environment (including particles of organic and biological origin: polymer nanoparticles, micro- and extracellular vesicles, liposomes, viruses etc).
\end{abstract}

Visualization of small nanoparticles is one of the basic challenges of the modern optical microscopy. Extremely low level of light signals makes this task very difficult. It is especially difficult to detect the so-called "invisible" nanoparticles, which weakly absorb a light of a visible band and are characterized by the index of refraction close to its value in nearby environment. Such objects are typical in biology media and are often used in modern technologies (for example, solution of polymer nanoparticles in water).

Despite the evident success of the fluorescence microscopy in high sensitive nanoparticle detection it has a set of fundamental shortcomings. First, a necessity of sample labelling that might alter properties of particles, and secondly, an undesirable photobleaching of fluorescence emitters and a fluorescence saturation, that limited the number of emitted photons.

We present the novel optical technique for high sensitive image of nearly invisible nanoparticles based an elastic scattering detection. Our approach uses the modified suffer plasmon assisted microscopy and waveguide technique that allows to increase significantly the efficiency of sample illumination. The original scheme of optical signal detection, sensitive to distortion of scattered light, allows to increase noticeably signal to noise ratio. The important advantage of our microscope is the possibility to detect the same particles simultaneously by two different methods. It can be realized by collecting light signals from two different sides of the sample. This way allows to raise the reliability of nanoparticle detection with the assumption that the origin of the experimental errors in two methods is different (see, e.g. Fig. 1 and Fig. 2).

\footnotetext{
* Corresponding author: vainer@isan.troitsk.ru
} 


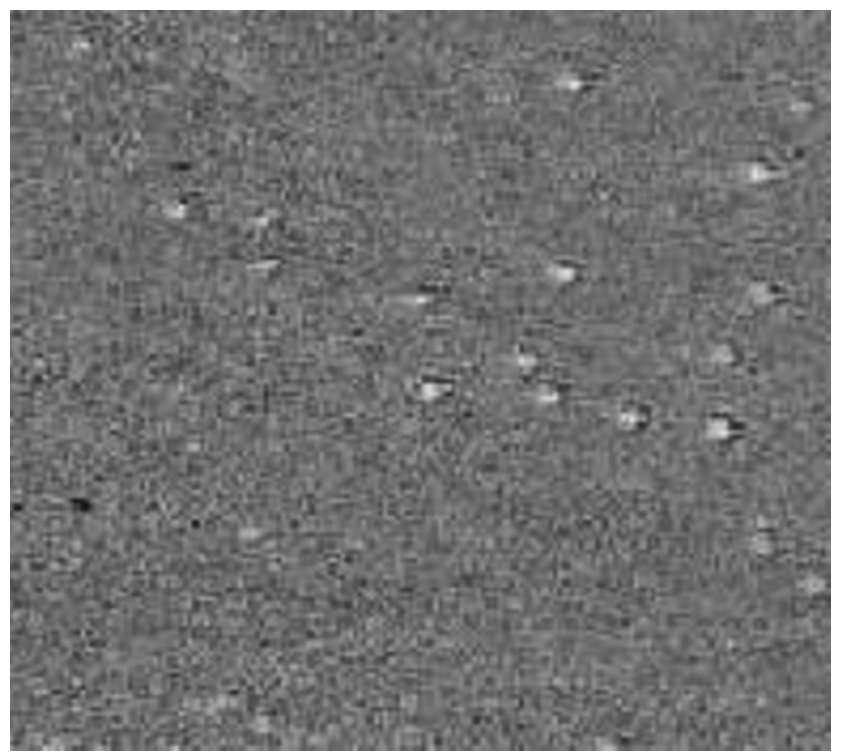

Fig. 1. Fragment of the detected image of polysterene nanospheres of $80 \mathrm{~nm}$ diameter.

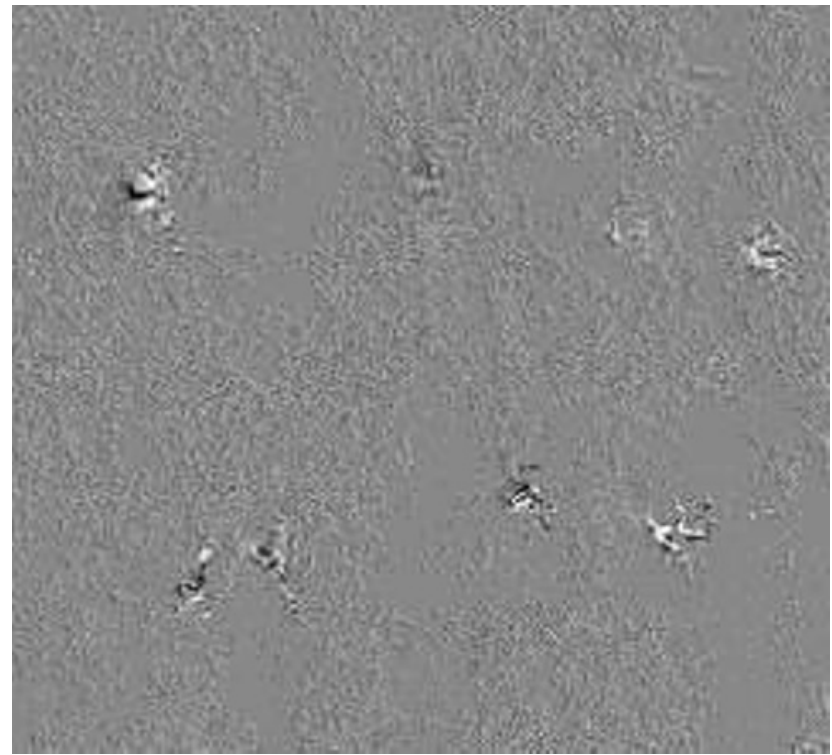

Fig. 2. Fragment of the detected image of liposomes of $\sim 100 \mathrm{~nm}$ diameter. 